\title{
Acoustic diagnosis of cavitation for centrifugal pumps of different materials.
}

\author{
Mostafa Swelam $^{1}$, Ashraf Kotb ${ }^{2}$, A.M. Abdulaziz ${ }^{3}$ \\ Department of Mechanical Power Engineering, Faculty of Engineering \\ Ain Shams University, Cairo, Egypt \\ ${ }^{1}$ Eng.Mostafaswelam@gmail.com $\quad-$ Tel. +20 1069030013 \\ ${ }^{2}$ Ashraf_Ibrahim@eng.asu.edu.eg - Tel. +20 1222481377

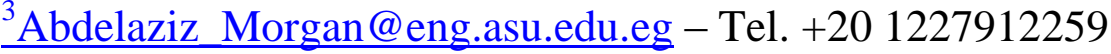

\begin{abstract}
Cavitation has destructive effects on pumps. It causes mechanical damage, decreases discharge and efficiency and increases noise and vibration. Therefore it must be detected during pump operation to find a way to eliminate it. This study concentrates on the effect of impeller material on cavitation and its effect on the acoustic emission level. Three different impeller materials are tested; stainless steel, brass and plastic. Cavitation is detected by analyzing sound spectrum. The experimental results show that the plastic impeller gives the lowest sound level compared to the stainless and the brass materials. It is also evident that the amplitudes of the high frequency range of sound spectrum are affected by the impeller material during normal operation of the pump. Cavitation results in further increase in the sound amplitudes in the high frequency range.
\end{abstract}

Keywords: Centrifugal pump; Cavitation; noise.

Nomenclature

BPF Blade pass frequency, $\mathrm{Hz}$

$c_{1} \quad$ Fluid velocity at impeller inlet, $\mathrm{m} / \mathrm{s}$

$c_{s} \quad$ Flow velocity at the pump suction , $\mathrm{m} / \mathrm{s}$

g Gravitational acceleration, $\mathrm{m} / \mathrm{s}^{2}$

$H \quad$ Pump total head, m 


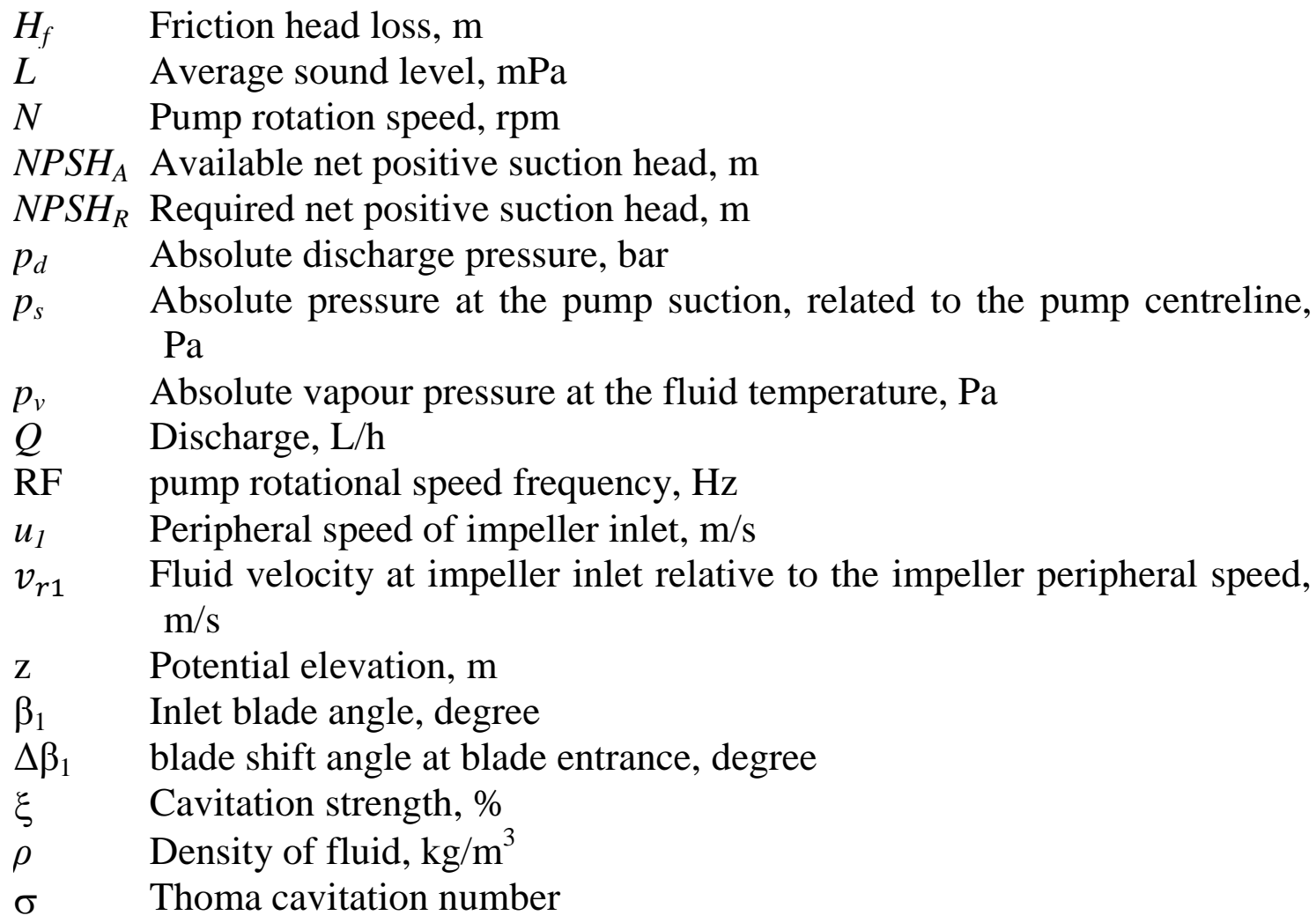

\section{Introduction}

It is expected that all pumps are subjected to cavitation. It happens when the static pressure at the suction port or through the impeller falls below the saturation pressure corresponds to the fluid temperature. Pressure is reduced due to many aspects; one of the inherent aspects is the inlet guide vanes [1].

Wherever the pressure falls, the fluid starts to flash and evaporate with the onset of bubbles where their number and size depend mainly on the value of pressure. Whenever the bubbles reach the high pressure point of the blade area, they pass through the fluid flow and collapse. The maximum amplitude of pressure variation at the leading edge of the blade for cavitation flow is 2.54 times larger than the corresponding non-cavitation flow because of the violent disturbances caused by cavitation shedding and explosion. [2].

Cavitation causes destructive consequences as results of pressure fluctuation, audible sound, and vibration. It is associated with a non-recoverable degradation of the hydraulic performance, impeller pitting, and material erosion. Therefore cavitation in pumps must be detected and prevented.

The incipient of cavitation is detected in various methods: determination of a net positive suction head at constant flow and velocity, visualization of the input flow on the impeller eye, paint erosion on impeller blades. Cavitation detection by acoustic measurements is based on understanding the sources of the generated noise from the pump. 
Hydraulic sources of noise include both the transient and instationarity of flow causes; Transients; during the startup and shutdown of the pump, opening or closing of servicing valves may cause severe pressure waves (water hammer) with high intensity of noise levels. Instationarity of flow; as a result of asymmetric outgoing flow of the impeller due to secondary flows in the rotating impeller, number of vanes and thickness which cause a notch in the wake flow, guide vanes and tongue in the pump casing. The instationarity of flow is a combination of both the effect of pressure pulsations and turbulence. Pressure pulsations depend on the design of impeller, diffuser, operating parameters, and the distance between impeller and the volute tongue. Turbulence induces vortices and wakes in the clearance between the impeller vane tips and the diffuser or volute lips. Pressure pulsations produced in this way impinge on the impeller and result in shaft vibrations and noise over a wide frequency range.

Also, the noise generated from the pump cavitation depends on the fluid temperature in the range from $80^{\circ} \mathrm{C}$ and $280^{\circ} \mathrm{C}$ for water [3]. In case of pump cavitation, the cavitation noise has a characteristic crashing or hissing noise, which sometimes overlays the blade by passing harmonics generated by the random, high frequency high energy band. It is well known but limited to the high frequency of ultrasound to detect cavitations in pumps (over $20 \mathrm{kHz}$ ), whereas noise can be measured inside the pump. The cavitation noise studies continue to be conducted in the audible frequency range (from $20 \mathrm{~Hz}$ to $20 \mathrm{kHz}$ ), with great attention paid to rotational frequency and harmonics.

For $5 \mathrm{~kW}$ pumping set with six semi-open impellers, Cudina found at $147 \mathrm{~Hz}$, there is 12 and $20 \mathrm{~dB}$ difference in the noise level between the incipient and fully developed cavitation, with recommendations of using the $147 \mathrm{~Hz}$ sound signal as a cavitation detector $[4,5]$.

For three pumping sets, centrifugal pump with six semi-open impellers, chemical centrifugal pump with six closed impellers and water mixed-flow pump with five semi-open rotor blades, Cudina and Prezelj measured the noise at discrete frequencies in the audible range to detect the incipient and fully developed cavitation in pump. They found great enough difference in the noise level between the two conditions for various distance between the microphone and the pump, with a great difference in noise peak at the discrete frequency of $147 \mathrm{~Hz}[6,7]$.

Two centrifugal pumps, the metal alloy one has six blades closed impeller and the plastic one has six blades semi-open impeller, Cernetic et al. found the discrete frequency for the plastic one at $148 \mathrm{~Hz}$ [8].

Closed impeller and semi-open impeller for two pumps, Cernetic tested two the different centrifugal pumps and found that; the convenient range of frequency for cavitation detection is above $1000 \mathrm{~Hz}$ [9].

The research in this field extends to consider different measuring techniques and instrumentation, V. Bull et al. used a fiber-optic hydrophone to detect the acoustic emissions over frequency range of $15-20 \mathrm{MHz}$ [10]. Antonio Baldassarre et al. used image processing for visual analysis of cavitation conditions [11]. 
Three different impellers of a laboratory centrifugal pump with a Plexiglas casing, using flow visualization, G. Mousmoulis, et al., measured the noise due to cavitation in different impellers and studied the effects of blade geometry in cavitation development, They found that the geometrical characteristics of the impeller affect cavitation development and behavior, while an acoustic emission sensor can be applied for successfully detecting the onset of this mechanism [12]. A centrifugal pump with controlling the inlet suction valve, Ahmed R. investigated the effect of various suction valve openings on cavitation in the pumps. He found that acoustic signal using different statistical features can provide a good indication to the beginning of cavitation in pump under different suction valve openings [13]. L. ALFAYEZ and D. MBA used applicability of the acoustic emission (AE) technique for determining the incipient cavitation and best efficiency point (BEP) of an operating pump, Results obtained demonstrated the successful use of the AE technique for detecting incipient of cavitation [14].

The main aim of the present work is measuring and analyzing the sound spectrum of a pump with three different impeller materials under cavitation and noncavitation conditions. It is an attempt to declare the validity of cavitation detection for different impellers and the further understanding of the effects of materials on cavitation development.

\section{Theoretical Background}

The static pressure along a stream line is governed by Bernoulli equation, Equation (1), where it decreases as a result of increasing the velocity.

Fig. 1 shows a schematic distribution of the pressure along the pump impeller where it has a minimum value somewhere at the impeller eye. Cavitation occurs when the pressure falls below the vapor pressure.

$$
\frac{p_{S}}{\rho g}+\frac{c_{S}^{2}}{2 g}+z+H=\text { Constant }
$$

Available net positive suction head $\left(\mathrm{NPSH}_{A}\right)$ is defined as the available total suction head at the pump inlet above the head corresponding to the vapor pressure at fluid temperature.

$$
N P S H_{A}=\frac{p_{s}-P_{V}}{\rho g}+Z-H_{f}+\frac{c_{S}^{2}}{2 g}
$$

$\mathrm{NPSH}_{R}$ equals $\mathrm{NPSH}_{A}$ when the pump head deteriorates by $3 \%$ from the main characteristic curve. Thoma cavitation number is defined by:

$$
\sigma=\frac{N P S H_{A}}{H}
$$

The total head is not constant when investigations into cavitation extend to various pump discharges. Therefore, during this study, the so called cavitation strength parameter, suggested by [15], is used:

$$
\xi=\frac{N P S H_{R}-N P S H_{A}}{N P S H_{R}} \%
$$

During cavitation, bubbles are formed in a low pressure media. As the fluid moves through the impeller blades, the pressure is increased by the amount of energy 
added. At a certain pressure level the bubbles collapse near the wall producing large range of sound spectrum which can be sensed by acoustic sensor in the time domain. Integration of the acoustic amplitude with time would give the overall sound level. To correlate sound levels with cavitation only, other factors must be eliminated.

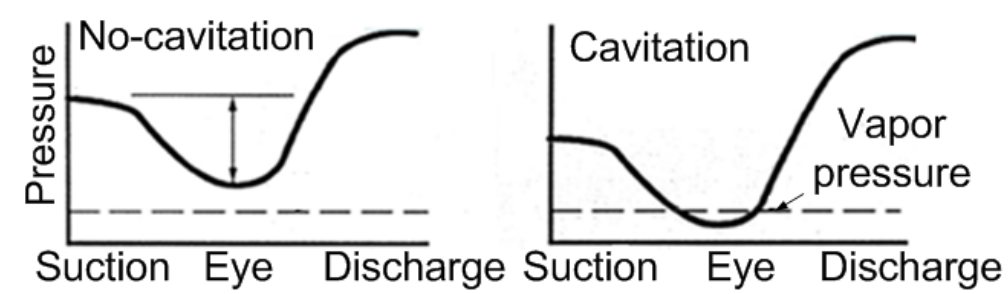

Fig. 1. Schematic distribution of the static pressure along the pump impeller.

\section{Materials and Methods}

The test rig is in accordance with ISO 3555 and BS EN ISO 9906/2000 [16] specifications for acceptance tests for centrifugal, mixed flow and axial pumps Class B tests is shown in Fig. 2(a), Fig. 2(b). It is a closed loop water circuit composed of a pump, a tank, connecting PVC pipes and regulating valves. The maximum head and pump discharge at $2850 \mathrm{rpm}$ are $46 \mathrm{~m}$ and $501 / \mathrm{min}$.

The pump is tested with three impellers of three different materials; brass, stainless steel and plastic. All impellers have six blades of backward curved type and closed impeller types. The outer and inner diameters are 125 and $36 \mathrm{~mm}$. The pump rotational speed is precisely controlled with an electronic inverter within $0.1 \mathrm{~Hz}$ resolution. The test rig is provided with a set of measuring instrumentation for different variables. Water flow rate is measured by a pre-calibrated electronic flow meter. The upstream and downstream pressures are measured by pre calibrated dial gauges with additional pressure transducer; Keller $\mathrm{Pa} 21 \mathrm{SR}$, to record the downstream pressure. Sound spectrum is measured with BK 4134 microphone. Signal is acquired through NI 9234 data acquisition card. The microphone is kept at fixed point of $50 \mathrm{~cm}$ apart from the pump and at the same pump level as shown in Fig. 2(c). All measurements are acquired to a PC via the very specialized NI 9234 data acquisition card. Lab view software is used to simultaneously record the mass flow rate, downstream pressure, and sound levels. The software performs Fast Fourier Transform analysis. The maximum uncertainty in measuring the mass flow rate as repeated 14 times for each test case is $0.5 \%$. The pressure transducer accuracy is \pm 0.5 of full scale. The BK 4134 microphone has a bandwidth of 5$20000 \mathrm{~Hz}$ with $\pm 1 \mathrm{db}$ in the range of $5-10 \mathrm{kHz}$. Data acquisition is performed by an NI 9234 card of 4 channels, 24 bits with amplifying capability. The sampling frequency is $52.2 \mathrm{kHz}$ for each channel. 
The measurements are repeated 30 times for the same run and the scheme of peakhold is applied. Meanwhile the instantaneous volume flow rate and the downstream pressure are continuously acquired and averaged for the 30 second run. All measurements are, therefore, taken simultaneously. The raw and processed data are then stored in an Excel file sheet.

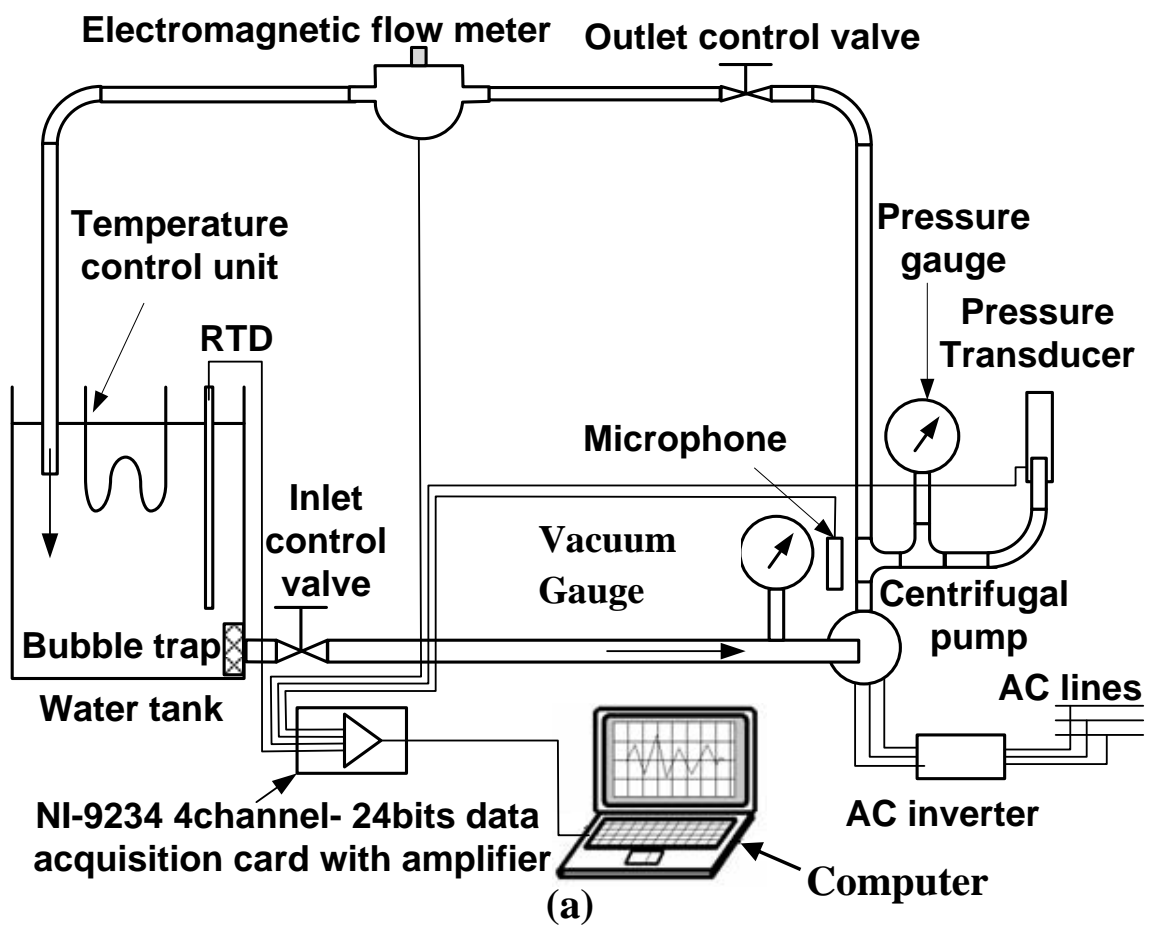

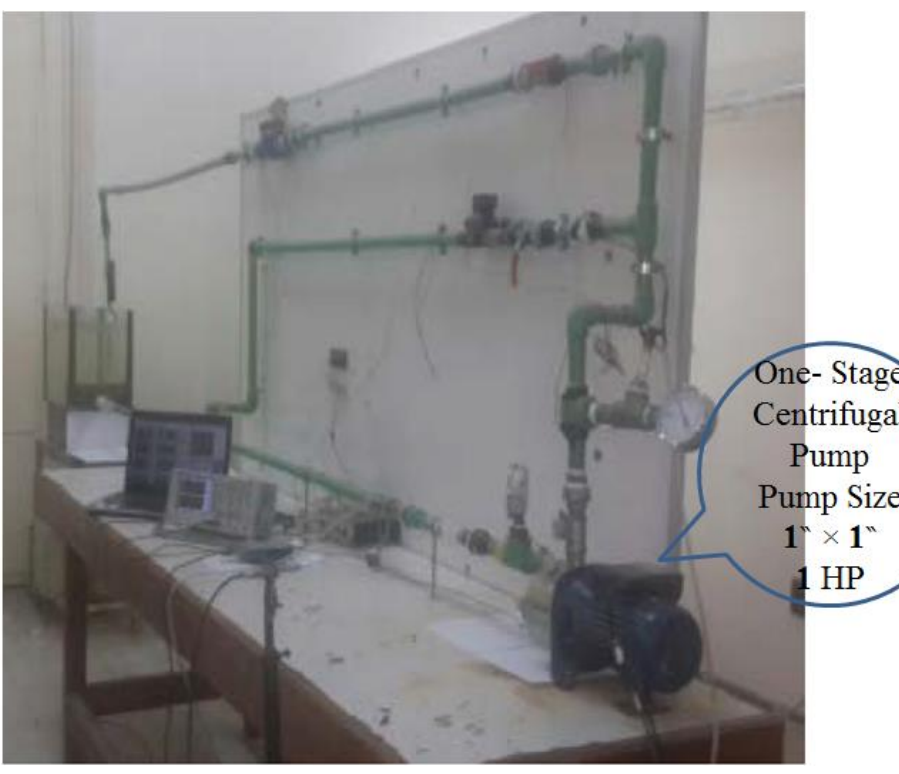

(b)

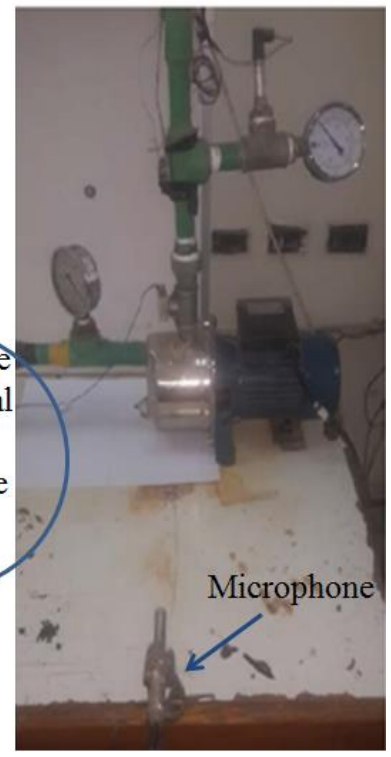

(c)

Fig. 2. (a) Experimental test rig, (b) Real picture of the test rig, (c) All location of the acoustic sensor relative to the pump. 


\section{Results and Discussion.}

The first part in this section utilizes the hydraulic characteristics of the centrifugal pump to investigate the inception and development of cavitation for three impellers with different material at constant rotational speed and variable suction pressure.

Fig. 3 shows the experimentally measured characteristic curves (discharge versus total head). They are almost identical for the three impellers at a rotational speed of $2850 \mathrm{rpm}(47.5 \mathrm{~Hz})$ and variable absolute suction pressure from 0.1 to $0.78 \mathrm{bar}$. It is illustrated that, there is a definite discharge at which the performance curve separates from the conventional profile and the head suddenly starts to deteriorate while the flow tends to be choked (independent on head). The working point at the beginning of the choked flow is defined as the separation point (practically when the head drops by $3 \%$ ).

The value of the available net positive suction head for the separation point is coincident with the required net positive suction head provided by the pump manufacturer as shown in Fig. 3. One can deduce that; the pump starts to face cavitation from the point at which the characteristic curve separates from the conventional one and beyond. Therefore the hydraulic performance may be considered as a first estimate for the inception of cavitation. Table 1 shows the characteristic of the separation points and the corresponding available net positive suction head for each suction pressure.

As an emphasis for the performance deterioration associated with the cavitation, Fig. 4 shows, experimentally measured efficiency curves versus the discharge at the same previous conditions. Increasing the pump discharge increases the efficiency till its maximum value just before reaching the separation flow rate and then the efficiency decreases sharply.

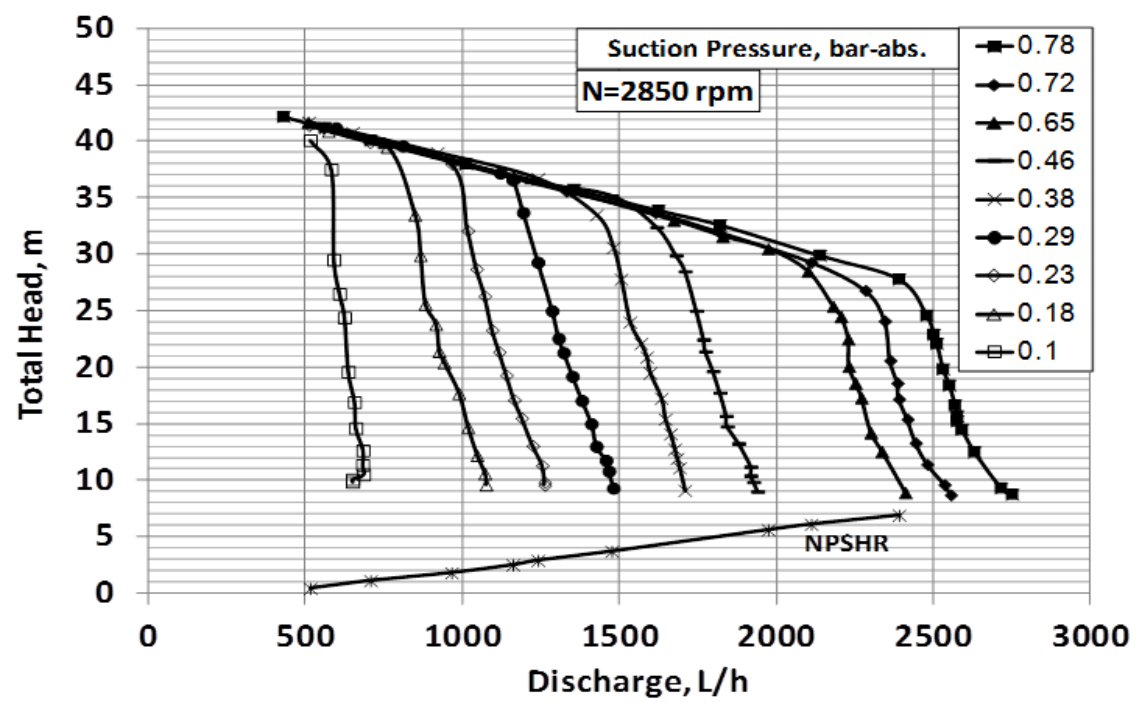

Fig. 3 Pump characteristics at variable suction pressures. 
Table 1. Separation point and NPSHA.

\begin{tabular}{|c|c|c|}
\hline Suction Pressure, bar (abs) & Discharge, L/hr & NPSHA. m \\
\hline 0.78 & 2390 & 6.71 \\
\hline 0.72 & 2110 & 6.25 \\
\hline 0.65 & 1970 & 5.6 \\
\hline 0.46 & 1475 & 3.87 \\
\hline 0.38 & 1240 & 3.13 \\
\hline 0.29 & 1160 & 2.23 \\
\hline 0.23 & 956 & 1.67 \\
\hline 0.18 & 707 & 1.21 \\
\hline 0.1 & 515 & 0.43 \\
\hline
\end{tabular}

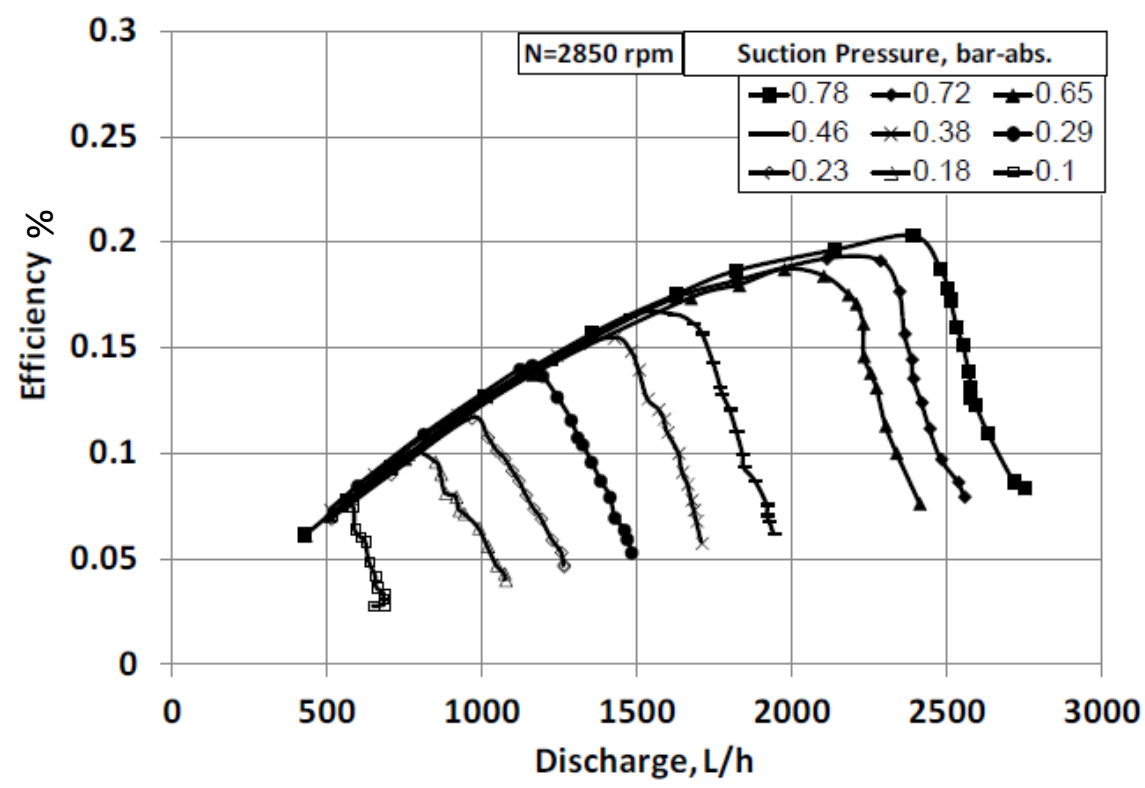

Fig. 4 Pump efficiency at variable suction pressures.

In the second part of this section, the emitted sound from the pump for each impeller is measured and analyzed during normal and cavitating operational conditions. Figures 5 (a) and (b) show experimentally the measured average sound level versus the pump discharge at rotational speed of $2850 \mathrm{rpm}(47.5 \mathrm{HZ})$ and two absolute values of suction pressure 0.78 bar and 0.65 bar for the plastic, brass and stainless steel impeller materials.

For all impeller at certain suction pressure, as the discharge increases the sound level slightly decreases, followed by a slight increase, then an abrupt decrease followed by an abrupt increase as shown by circles in Fig. 5 (a) and (b). The first sound minimum value expresses the best operating condition where the flow 
velocity tangentially passes the pump blades. As the discharge increases beyond this point the flow is no longer tangential to the blades and cavitation occurs due to flow circulation (cavitation zone). The sound noise, however, has a second minimum value at the cavitation zone which is much lower than the first one.

The most valuable result is that the plastic impeller produces the least average noise level as compared to the brass and stainless impellers.

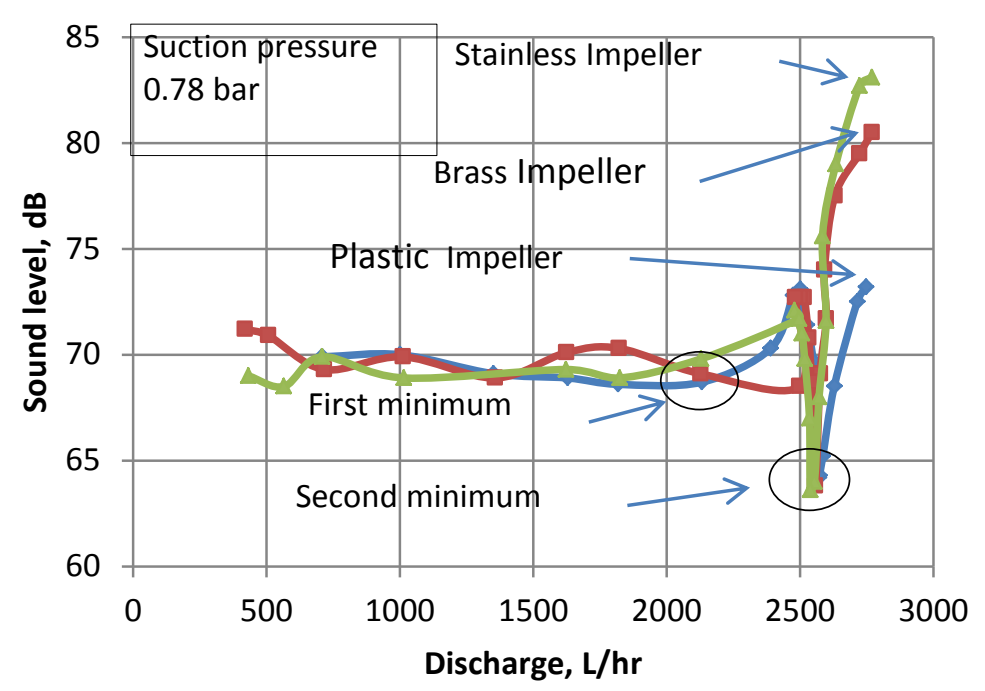

Fig. 5-a. Overall Sound level at 0.78 bar suction pressure.

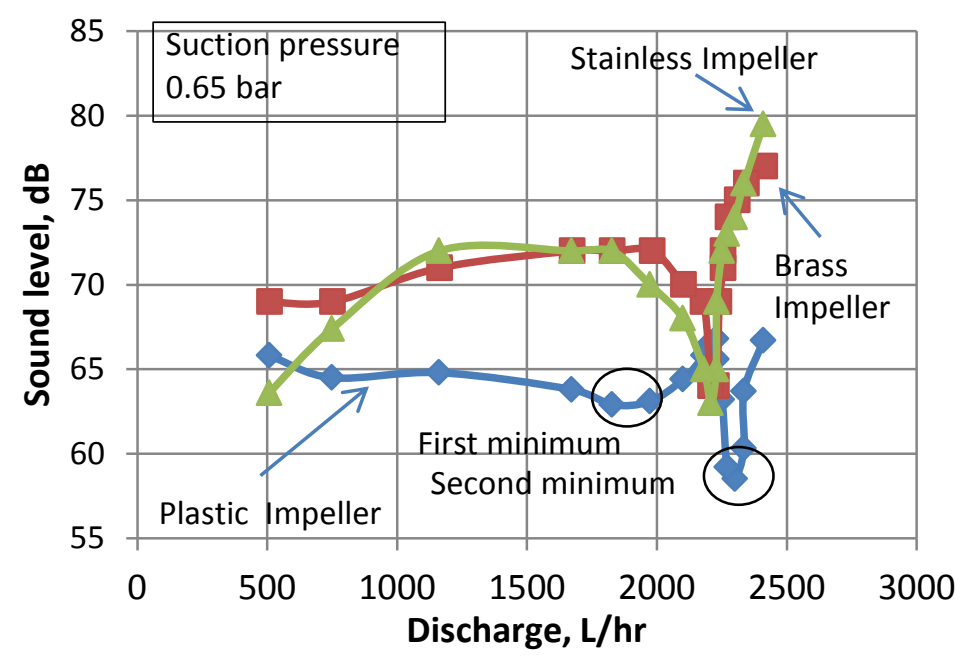

Fig. 5-b Overall Sound level at 0.65 bar suction pressure.

Analysis of sound spectrum emitted from the pump during operation using Fast Fourier Transform for frequency range 10-20000 Hz is shown in Figs. 6 \&7 for the three impellers at suction pressure 0.87 and 0.65 bar. Three cases of no-cavitation (a), inception of cavitation (b) and cavitation (c) are shown in each figure. Overall 
view the effective frequency range lie between 1000 and $13000 \mathrm{~Hz}$. There is peak amplitude around the frequency $3400 \mathrm{~Hz}$. The sound data points, around this frequency, are more or less normally distributed. Other active amplitudes are also recorded around the frequency $12000 \mathrm{~Hz}$. The average sound amplitude, denoted by the letter L, is taken as a measure for sound intensity when comparing emitted sound from each impeller. It can be seen from both figures that the plastic impeller emits the least average sound level as compared to the brass and stainless steel impellers. It is also evident that cavitation increases the sound level (cases b and $\mathrm{c}$ compared to case a). For instance for the plastic impeller at 0.78 bar suction pressure, the maximum sound amplitudes are $2.04,3.2$ and $3.7 \mathrm{mPa}$ for the three cases of no-cavitation, inception of cavitation and cavitation. These results agree with the findings of Cernetic [9] who tested two the different centrifugal pumps, one is made of metal alloy and the other is made of plastic material and found that; the convenient range of frequency for cavitation detection is above $1000 \mathrm{~Hz}$. This reflects that the maximum amplitudes of the discrete frequencies through the whole range of sound spectrum increases with cavitation. 


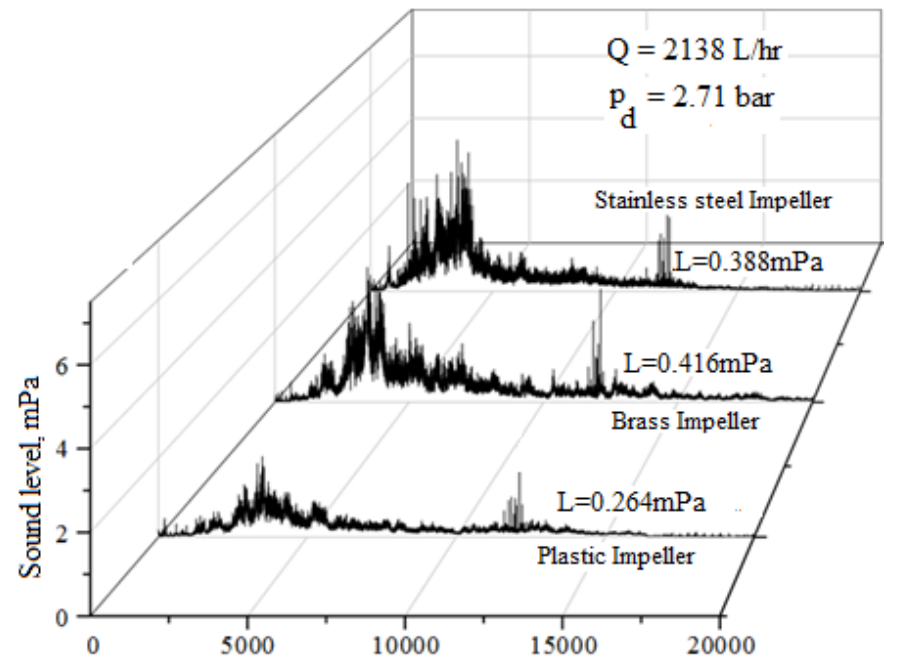

(a)

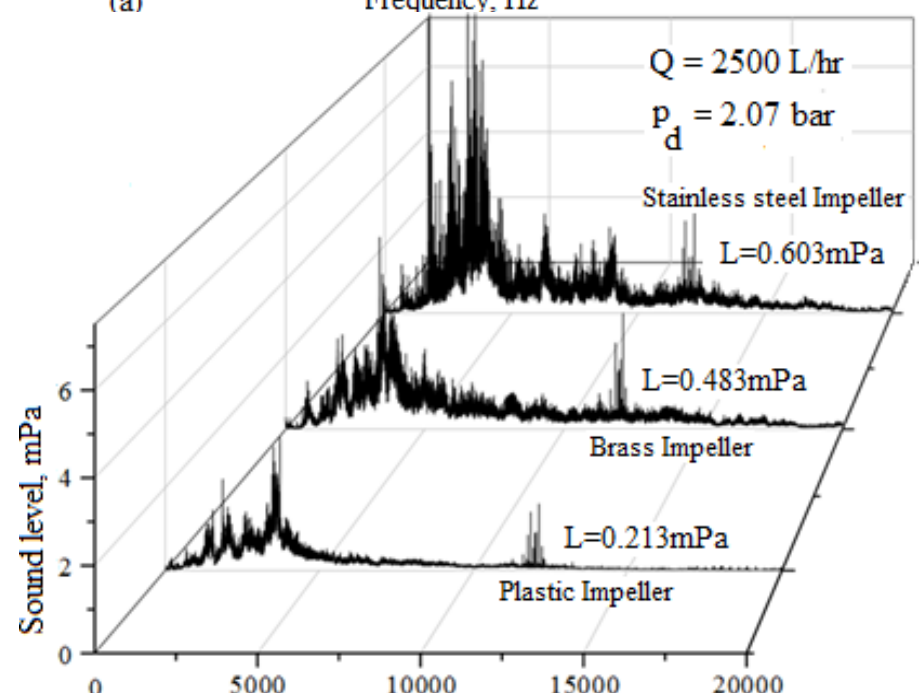

(b)

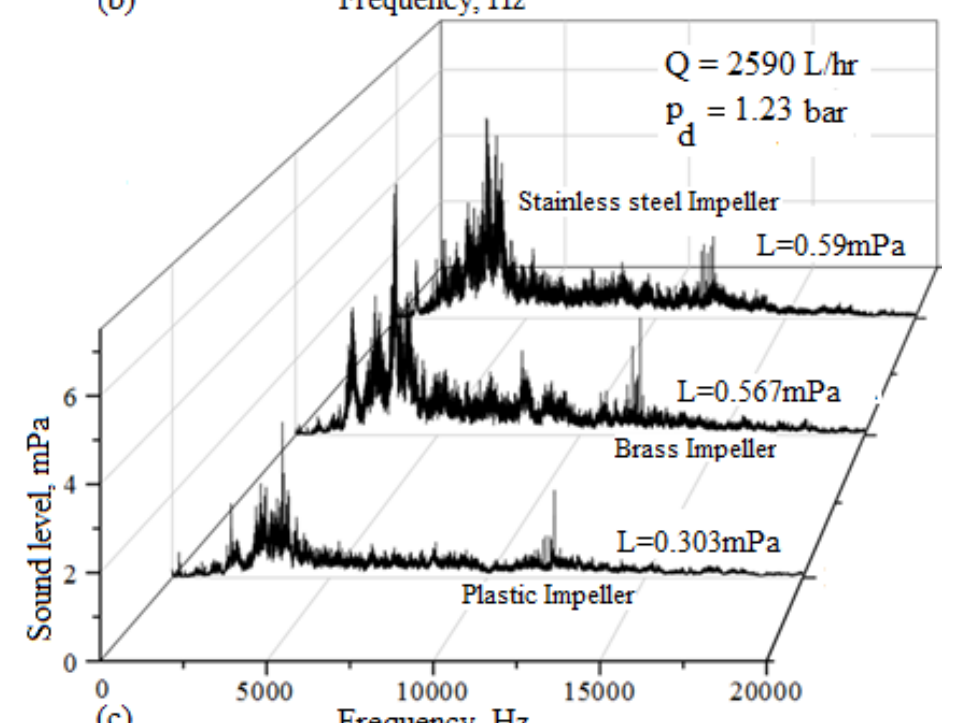

(c) Frequency, $\mathrm{Hz}$

Fig. 6 Sound level spectrums for: (a) no-cavitation, (b) incipient of cavitation and (c) cavitation cases at suction pressure 0.78 bar for three different impellers. 


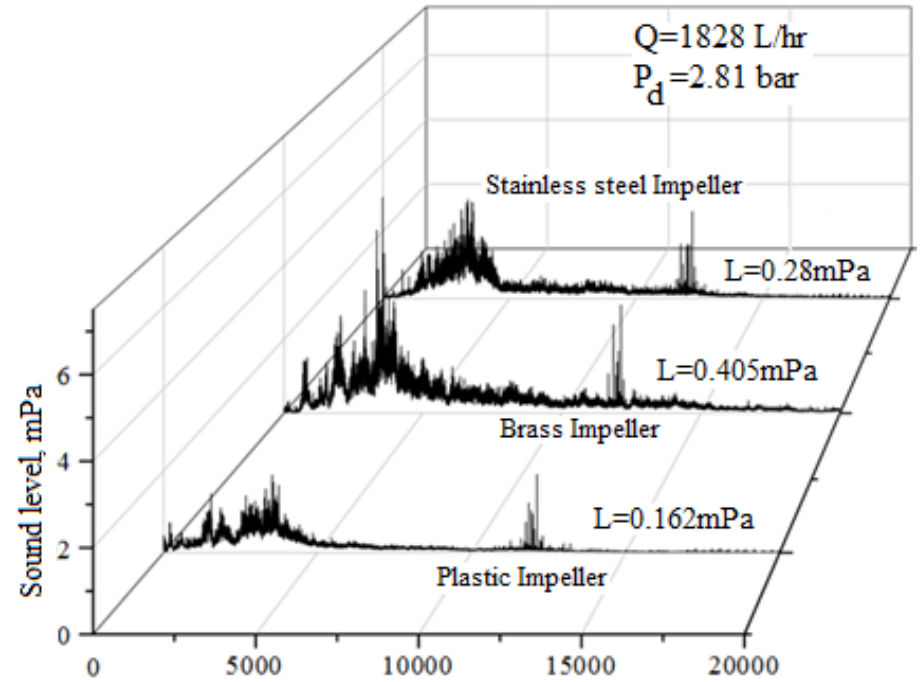

(a)

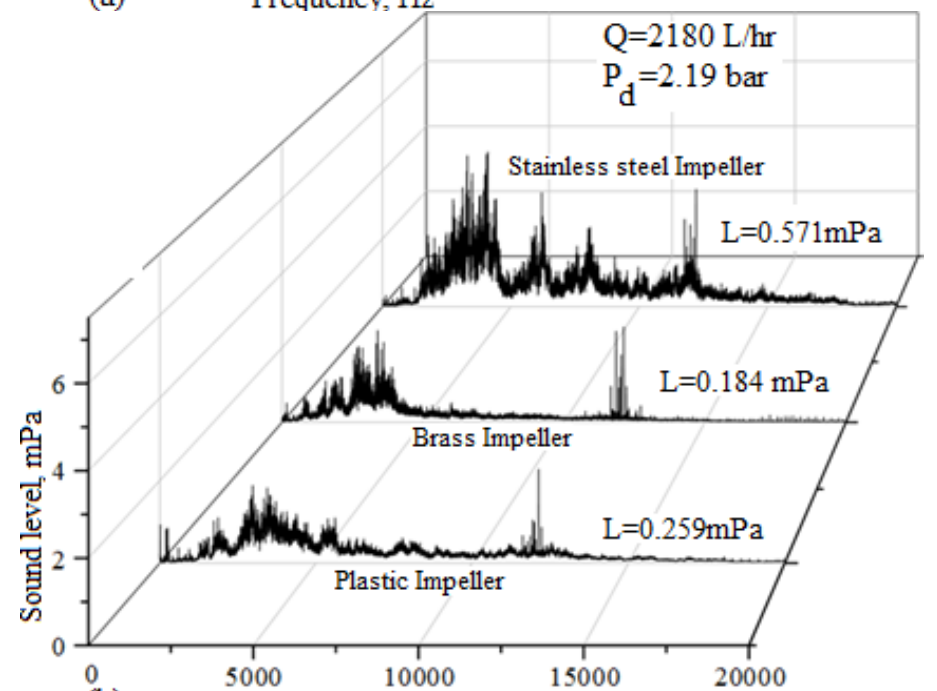

(b)

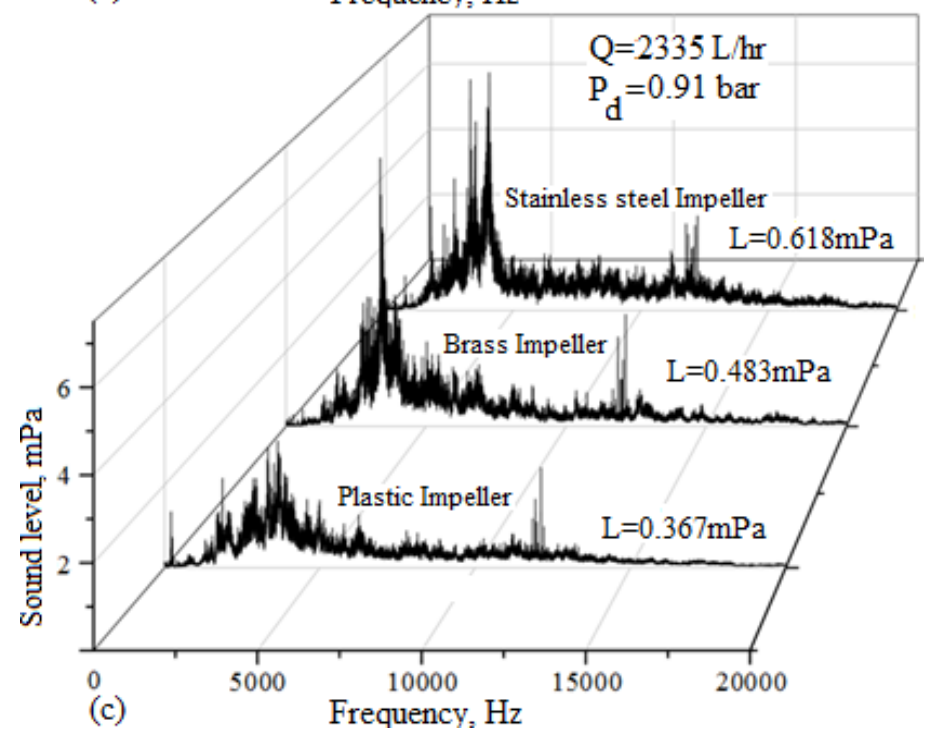

Fig. 7 Sound level spectrums for: (a) no-cavitation, (b) incipient of cavitation and (c) cavitation cases at suction pressure 0.65 bar for three different impellers. 


\section{Conclusions}

This study discusses the inception and development of cavitation in three different impellers of a centrifugal pump at fixed speed of $2850 \mathrm{rpm}$ and variable absolute values of suction pressures from 10 to $78 \mathrm{kPa}$, and acoustic emissions monitoring, the following remarks could be concluded:

1- Plastic impeller produces the least sound noise as compared to the brass and stainless steel impellers.

2- Average sound level varies with pump flow rate and cannot be used alone for cavitation detection.

3- Maximum discrete sound amplitude occurs around a frequency of $3500 \mathrm{~Hz}$.

4- Cavitation increases the amplitude of sound spectrum at the maximum discrete frequency.

5- Maximum discrete sound amplitude is more promising way for detection of cavitation in pumps.

\section{References}

[1] Tan, L., Zhu, B.S., Cao, S.L., et al., "Influence of Prewhirl Regulation by Inlet Guide Vanes on Cavitation Performance of a Centrifugal Pump", Energies, 2014, 7, 1050-1065. http://dx.doi.org/10.3390/en7021050.

[2] Tan, L., Zhu, B.S., Cao, S.L., et al., "Numerical Simulation of Unsteady Cavitation Flow in a Centrifugal Pump at Off-Design Conditions", Proceedings of the Institution of Mechanical Engineers, Part C: Journal of Mechanical Engineering Science, 2014, Vol 228, 1994-2006. http://dx.doi.org/10.1177/0954406213514573.

[3] Kudirka, A.A. and DeCoster, M.A., "Jet Pump Cavitation with Ambient and High Temperature Water. Journal of Fluids Engineering: 1979, 101, 93-99. http://dx.doi.org/10.1115/1.3448741.

[4] Cudina, M., "Detection of Cavitation Phenomenon in a Centrifugal Pump Using Audible Sound", Mechanical Systems and Signal Processing, 2003, 17, 1335-1347. http://dx.doi.org/10.1006/mssp.2002.1514.

[5] Cudina, M., "Noise as an Indicator of Cavitation in a Centrifugal Pump. Acoustical Physics, 49, 463-474. From Akusticheski Ï Zhurnal", 2003, 49, 551-564.

[6] Cudina, M. and Prezelj, J., "Detection of Cavitation in Operation of Kinetic Pumps. Use of Discrete Frequenc Tone in Audible Spectra. Applied Acoustics", 2009, 70, 540-546. http://dx.doi.org/10.1016/j.apacoust.2008.07.005.

[7] Cudina, M. and Prezelj, J., "Detection of Cavitation in Situ Operation of Kinetic Pumps: Effect of Cavitation on the Characteristic Discrete Frequency Component. 
Applied Acoustics Applied Acoustics", 2009, 70, 1175-1182. http://dx.doi.org/10.1016/j.apacoust.2009.04.001.

[8] Cernetic, J., Prezelj, J. and Cudina, M., "Use of Noise and Vibration Signal for Detection and Monitoring of Cavitation in Kinetic Pumps". The Journal of the Acoustical Society of America, 2008, 123, 3316. http://dx.doi.org/10.1121/1.2933777.

[9] Cernetic, J., "The Use of Noise and Vibration Signals for Detecting Cavitation in Kinetic Pumps", Proceedings of the Institution of Mechanical Engineers, Part C: Journal of Mechanical Engineering Science, 2009, 223, 1645-1655. http://dx.doi.org/10.1243/09544062JMES1404.

[10] Bull, V., Civale, J., Rivens, I. and ter Haar, G.R., "Cavitation Detection Using a Fibre Optic Hydrophone: A Pilot Study", AIP Conference Proceedings, 2011, 1359, 85. http://dx.doi.org/10.1063/1.3607887.

[11] Baldassarre A., De Lucia, M. and Nesi, P., "Real-Time Detection of Cavitation for Hydraulic Turbomachines", Real-Time Imaging, 1998, 4, 403-416.

[12] G. Mousmoulis, et al., "Experimental analysis of cavitation in a centrifugal pump using acoustic emission", Vibration measurements and flow visualization, European Journal of Mechanics / B Fluids (2018), https://doi.org/10.1016/j.euromechflu.2018.10.015.

[13] Ahmed R., "Experimental Investigation of the Effect of Suction Valve Opening on the Performance and Detection of Cavitation in the Centrifugal Pump Based on Acoustic Analysis Technique", Department of Mechanical Engineering Dep. Mustansiriyah University, 2018, https://doi.org/10.24425/aoa.2019.126352.

[14] L. Alfayez and D. Mba., "Detection of incipient cavitation and best efficiency point in a $2.2 \mathrm{mw}$ centrifucal pump using acoustic emission", School of Mechanical Engineering, Cranfield University 2004.

[15] A. M. Abdulaziz \& Ashraf Kotb, "Detection of pump cavitation by vibration signature, Australian Journal of Mechanical Engineering", 2015, ISSN: 1448-4846 (Print) 2204-2253.

[16] BS 5316-2:1977, ISO 3555:1977 and BS EN ISO 9906:2000, "Specification for Acceptance Tests for Centrifugal, Mixed Flow and Axial Pumps", Class B Tests, MCE/6. BSI. 